\title{
GLOBAL-BETA MEASUREMENT AND CORRECTION AT THE KEKB RINGS
}

\author{
A. Morita, H. Koiso, K. Oide, Y. Ohnishi, KEK, 1-1 Oho, Tsukuba, Ibaraki 305-0801, Japan
}

\section{Abstract}

The global-beta correction is one of the optics corrections such as dispersion and xy-coupling corrections to realize a model lattice [1]. In the KEKB B-Factory, it is performed to regularize the ring optics for achieving high luminosity. The global-beta function is measured by reconstructing responses of orbits generated by 6 different steering magnets per plane. The correction of the beta function and the phase advance is performed by using the fudge factors of power supplies of quadrupole magnets. This correction scheme has been successfully. In the typical case, the rms of the beta function beat and the betatron tune difference are corrected within $5 \%$ and 0.0005 , respectively. In the luminosity run, we can operate the low energy ring(LER) with the horizontal betatron tune very close to half-integer(45.5050). In this paper, we will report in detail the measurement and correction techniques and its performance in the KEKB operation.

\section{GLOBAL-BETA MEASUREMENT}

In our measurement method, we reconstruct both beta function $\beta(s)$ and betatron phase advance $\phi(s)$ from the set of the closed orbit distortions(CODs) generated by the single steering kick. From the first order perturbation theory of the ring, the displacement of the closed orbit is given as

$$
\begin{array}{r}
\Delta \chi(s)=\frac{\sqrt{\beta_{\chi}(s)}}{2 \sin \pi \nu_{\chi}} \int_{C} \Delta \theta_{\chi}\left(s^{\prime}\right) \sqrt{\beta_{\chi}\left(s^{\prime}\right)} \\
\cos \left(\left|\phi_{\chi}(s)-\phi_{\chi}\left(s^{\prime}\right)\right|-\pi \nu_{\chi}\right) d s^{\prime},
\end{array}
$$

where $\triangle \theta_{\chi}\left(s^{\prime}\right)$ is the distribution of the $\chi$ direction kick and $\int_{C}$ means the integration over one revolution of the ring. The direction symbol $\chi$ represents either $x$ or $y$ direction.

In the KEKB ring, we can observe both the betatron tune $\nu_{\chi}$ and the COD $\chi(s)$ at the beam position monitors(BPMs). Assuming that the steering does not overlap with the BPMs, the COD at $i$-th BPM generated by $j$-th steering is obtained as

$$
\triangle \chi_{i}^{j}=\frac{\triangle \theta_{j}}{2 \sin \pi \nu} \sqrt{\beta_{i}^{M}} \sqrt{\beta_{j}^{S}} \cos \left(\left|\phi_{i}^{M}-\phi_{j}^{S}\right|-\pi \nu\right),
$$

where superscripts ${ }^{M}$ and ${ }^{S}$ indicate the BPM and the steering, respectively. $\triangle \theta_{j}$ is the kick angle by $j$-th steering. The sign of $\phi_{i}^{M}-\phi_{j}^{S}$ is given by the geometry of the ring, because the betatron phase advance is monotonic.
For convenience, we introduce following notations:

$$
\begin{gathered}
f_{j}=\frac{\triangle \theta_{j}}{2 \sin \pi \nu}, S_{i j}=\operatorname{sign}\left(\phi_{i}^{M}-\phi_{j}^{S}\right), \\
X_{i}^{l}=\sqrt{\beta_{i}^{l}} \cos \phi_{i}^{l}, Y_{i}^{l}=\sqrt{\beta_{i}^{l}} \sin \phi_{i}^{l} \quad(l=M, S) .
\end{gathered}
$$

Using these notations, Eq. 2 is rewritten as follows:

$$
\begin{aligned}
\triangle \chi_{i}^{j} & =\left(\cos \pi \nu f_{j} X_{j}^{S}-S_{i j} \sin \pi \nu f_{j} Y_{j}^{S}\right) X_{i}^{M} \\
& +\left(\cos \pi \nu f_{j} Y_{j}^{S}+S_{i j} \sin \pi \nu f_{j} X_{j}^{S}\right) Y_{i}^{M} \\
& =\left(\cos \pi \nu X_{i}^{M}+S_{i j} \sin \pi \nu Y_{i}^{M}\right) f_{j} X_{j}^{S} \\
& +\left(\cos \pi \nu Y_{i}^{M}-S_{i j} \sin \pi \nu X_{i}^{M}\right) f_{j} Y_{j}^{S} .
\end{aligned}
$$

To obtain the beta function, we fit Eq. 5-6 to the set of the measured closed orbits by using the least square method. The residual error sum of squares looks like the quadratic form of either $\left\{X_{i}^{M}, Y_{i}^{M}\right\}$ or $\left\{X_{j}^{S}, Y_{j}^{S}\right\}$ coordinates. Thus, the residual error is easily improved by interleaving two types of the linear fit as follows. At the first step, the temporary $\left\{X_{i}^{M}, Y_{i}^{M}\right\}$ is given by using the model optics adjusted with the measured betatron tune. At the second step, $\left\{X_{j}^{\prime S}, Y_{j}^{\prime S}\right\}$ is calculated from given $\left\{X_{i}^{M}, Y_{i}^{M}\right\}$ by using the least square fit against Eq. 6. At the third step, improved $\left\{X_{i}^{\prime M}, Y_{i}^{M}\right\}$ is obtained from calculated $\left\{X_{j}^{\prime S}, Y_{j}^{\prime S}\right\}$ by using the least square fit against Eq. 5. Both second and third step are iterated until the residual error sum is converged. To stabilize the iteration method, the normalization of both $\beta(s)$ and $\phi(s)$ is requited, because the fitting residual of Eq. 2 is only determined by both the beta function product and the betatron phase difference. In order to normalize $\left\{X_{i}, Y_{i}\right\}$, we scale $\left\{X_{i}, Y_{i}\right\}$ after each fitting step to keep absolute norm: $\sum\left(\left|X_{i}\right|+\left|Y_{i}\right|\right)$.

This iteration method should be converged because of monotone decreasing of the residual error sum of squares, but the convergence speed is very slow. The minimum point obtained as the limit of our iteration method is the fixed point of the mapping defined by one iteration loop. In the practical use, we use the Newton-Raphson method with the numerical differential to find the fixed point of the mapping from $\left\{X_{j}^{S}, Y_{j}^{S}\right\}$ to $\left\{X_{j}^{\prime S}, Y_{j}^{\prime S}\right\}$, because the length of the steering side vector is shorter than the length of the BPM side vector.

\section{GLOBAL-BETA CORRECTION}

Assuming that the measured optics distortion is caused by the gradient field error of the quadrupole field, we try to correct the optics distortion by tuning the quadrupole field using the amplitude fudge factor (AF) of the quadrupole 
magnet power supply. Although the major source of the optical deformation is the alignment offsets of the sextupoles, they can be correctable by the nearby quadrupole settings. The beta function distortion by the gradient field error is given as

$$
\begin{aligned}
\frac{\triangle \beta_{\chi}(s)}{\beta_{\chi}(s)}=- & \frac{1}{2 \sin 2 \pi \nu_{\chi}} \int_{C} \triangle K_{\chi}\left(s^{\prime}\right) \beta_{\chi}\left(s^{\prime}\right) \\
& \cos \left(2\left|\phi_{\chi}(s)-\phi_{\chi}\left(s^{\prime}\right)\right|-2 \pi \nu_{\chi}\right) d s^{\prime}
\end{aligned}
$$

where $\triangle K_{\chi}\left(s^{\prime}\right)$ is an error distribution of gradient field in the $\chi$ plane. In the term of the quadrupole field strength $\left(K_{1} \equiv \frac{1}{B \rho} \frac{\partial B_{y}}{\partial x}\right), \triangle K_{x}\left(s^{\prime}\right)$ and $\triangle K_{y}\left(s^{\prime}\right)$ are described as $\triangle K_{1}\left(s^{\prime}\right)$ and $-\triangle K_{1}\left(s^{\prime}\right)$, respectively. The phase modulation and the tune shift are given by following integral forms

$$
\begin{gathered}
\triangle \phi_{\chi}(s)=\int_{0}^{s}\left(\frac{1}{\beta_{\chi}\left(s^{\prime}\right)+\triangle \beta_{\chi}\left(s^{\prime}\right)}-\frac{1}{\beta_{\chi}\left(s^{\prime}\right)}\right) d s^{\prime} \\
=\frac{1}{2 \sin 2 \pi \nu_{\chi}} \int_{C} \triangle K_{\chi}\left(s^{\prime}\right) \beta_{\chi}\left(s^{\prime}\right) \\
\\
\quad\left\{2 \sin 2 \pi \nu_{\chi} \sin ^{2} \min \left(\phi_{\chi}(s)-\phi_{\chi}\left(s^{\prime}\right), 0\right)\right. \\
\left.\quad+\sin \phi_{\chi}(s) \cos \left(2 \phi_{\chi}\left(s^{\prime}\right)-\phi_{\chi}(s)-2 \pi \nu_{\chi}\right)\right\} \\
\triangle \nu_{\chi}=\frac{1}{4 \pi} \int_{C} \triangle K_{\chi}\left(s^{\prime}\right) \beta_{\chi}\left(s^{\prime}\right) d s^{\prime} .
\end{gathered}
$$

The perturbation of the gradient field is described by using the amplitude fudge factor for $i$-th quadrupole family $\left(A F^{i}\right)$ as follows

$$
\triangle K_{1}^{i}(s)=K_{1}^{i}(s) \triangle A F^{i}, \triangle A F^{i} \equiv A F^{i}-1,
$$

where $K_{1}^{i}(s)$ is the designed field distribution of $i$-th quadrupole. Integrating Eq. 7-9 under Eq. 10, the response matrix from the quadrupole family $\triangle A F^{i}$ to the optics functions $\triangle \beta(s) / \beta(s), \triangle \phi(s)$ and $\triangle \nu$ is obtained. In order to determine the correction fudge factor, we minimize the following residual error sum of squares constructed by the beta function, phase advance and betatron tune of the model optics and the measurement

$$
\begin{aligned}
e^{2} & \equiv\left(\frac{\pi}{\sin 2 \pi \nu}\right)^{2}\left|\Delta \nu-\left(\nu^{\text {measured }}-\nu^{\text {model }}\right)\right|^{2} \\
& +\sum\left(\left|\frac{\triangle \beta(s)}{\beta(s)}-\frac{\beta^{\text {measured }}(s)-\beta^{\text {model }}(s)}{\beta^{\text {model }}(s)}\right|^{2}\right. \\
& \left.+\left|\triangle \phi(s)-\left(\phi^{\text {measured }}(s)-\phi^{\text {model }}(s)\right)\right|^{2}\right)
\end{aligned}
$$

where $\sum$ means the sum over the measured points. The fudge factor $\triangle A F^{i}$ minimizing Eq. 11 is obtained by the Singular Value Decomposition(SVD) of the response matrix generated from Eq. 7-10. The obtained fudge factor shows the correction factor for fitting the model optics to the measured one. Thus, the optics error can be compensated by updating the fudge factor of the quadrupole magnet power supply using following procedure

$$
A F_{n e w}^{i}=\frac{A F_{o l d}^{i}}{1+\triangle A F^{i}} .
$$

In Eq. 11, the equal weighting is applied to the individual residual error squares. If the variance of the measured value is known, the individual residual error square in Eq. 11 should be multiplied by the inverse square of the standard deviation. In the actual use, the extra weighting factor is applied in order to correct preferentially the specific optics parameter (ex. betatron tune, beta function at interaction region).

For using the global-beta measurement described in the previous section, we must pay attention to the measurement uncertainty. Our measurement method can not determine both the scaling factor of the beta function and the origin of the phase advance, because Eq. 2 is conserved by either the phase origin change or the beta function scale change keeping $\beta_{i}^{M} \beta_{j}^{S}$. If the optics distortion is enough small, such uncertainty is canceled out by normalization using model optics. In another case, this uncertainty problem can be avoided by either introducing the fudge factor for the beta amplitude and the phase origin to Eq. 2 as the unknown quantity or constructing the residual error sum of squares from the relative value between the measurement positions.

\section{MEASUREMENT AND CORRECTION AT THE KEKB RINGS}

In our site, the global-beta measurement and correction method shown in the previous sections are implemented as an automation tool on SAD[2], which has the script interpreter including accelerator model, EPICS[3] channel access and GUI toolkit based on Tcl/Tk[4]. Using such automation tool, we correct the ring optics after every regular maintenance whose interval is normally two weeks. In the following subsections, we present the detail of our implementation and the performance of our correction system.

\section{Measurement at the KEKB Rings}

In the actual measurement at the KEKB rings, we use 6 steerings for the horizontal beta measurement and 6 steerings for the vertical beta measurement. For avoiding the degeneration of the measured COD set, the betatron phases of the steering magnets are spreaded out. We exclude the horizontal steering among the dispersive section for avoiding the momentum shift. As the KEKB proper condition, the $2.5 \pi$ cell structure with non-interleaved chromaticity correction is used for arc cells[5]. In this structure, the sextupole pairs are placed without overlapping. The transfer matrix between sextupole pair is adjusted to $-\boldsymbol{I}^{\prime}$, which is a negative identical 4 by 4 transformation except the $-\boldsymbol{I}_{12}^{\prime}$ and $-\boldsymbol{I}_{34}^{\prime}$ components, in order to cancel out the quadrupole field generation by the closed orbit distortion propagated from outside of the sextupole pair. Therefore, we can not use the steering magnet between the sextupole pair for the measurement, because the single steering kick between the sextupole pair makes the serious tune shift and the optics distortion.

For keeping the amplitude of the COD, the steering kick 


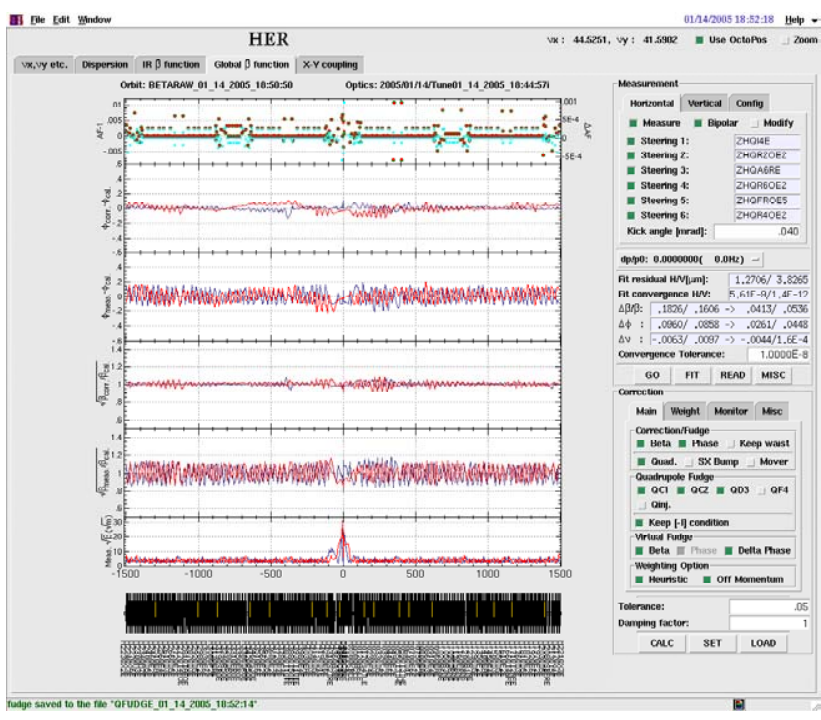

(a)Measurement before Correction

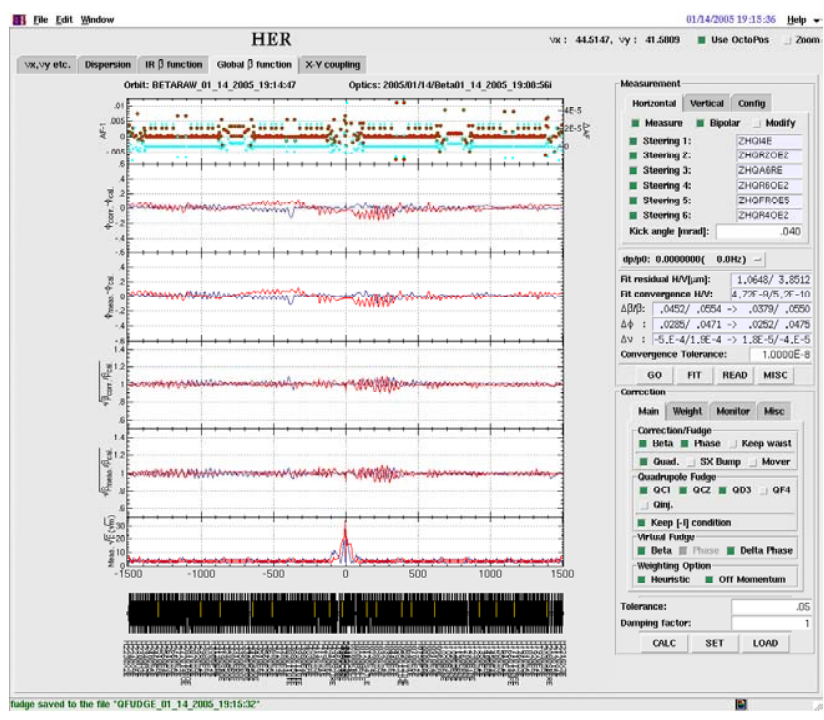

(b)Measurement after 2nd Correction

Figure 1: Measurement results before(a) and after(b) correction. In 1st column of graphs, current and desired values of AF are shown by green and red points, respectively. The measured value of phase advance error and square root ration of beta function are shown in 3rd and 5th column, respectively. 2nd and 4th column shows the prediction after this correction. In these graphs, blue and red lines correspond with horizontal and vertical optics function, respectively.

angle is scaled by the square root of the beta function at the steering magnet. The typical kick angle for the COD measurement is about $40 \mu \mathrm{rad}$ for $30 \mathrm{~m}$ beta function. The typical COD amplitude at the arc section is about $0.5 \mathrm{~mm}$. The iteration of the Newton-Raphson method in the beta function fitting is normally converged until 4 iterations. The typical rms of the residual error of the COD fitting is about $1 \sim 2 \mu \mathrm{m}$ except the vertical measurement of the High Energy Ring(HER). The typical rms of the vertical fitting residual at the HER is about $4 \mu \mathrm{m}$.

\section{Correction at the KEKB Rings}

In our global-beta correction, the quadrupoles related with $-\boldsymbol{I}^{\prime}$ arc cell condition and the quadrupole at the injection point are excluded from the corrector quadrupoles. In the usual correction, we apply 10 times extra weighting for tune errors in Eq. 11 and 0.05 is used as the relative tolerance for truncating small singular values of the SVD of the response matrix. In the usual case such as a correction after regular maintenances, the beta correction is normally converged until 3 iterations. After corrections, the typical residual error of the beta function $\triangle \beta / \beta$ is about $5 \%$ in $\mathrm{rms}$ and the betatron tune is corrected into $\pm 5 \times 10^{-4}$. In the present KEKB rings, the amplitude fudges of mostly quadrupoles are distributed within $\pm 5 \times 10^{-3}$. In the sequence of the beta corrections at the KEKB ring startup process, the typical range of the displacement of the fudge factor is $\pm 2 \times 10^{-4}$.

As an extra corrector, we can use a cosine like bump around sextupole pair. By making the same directional displacement of the horizontal orbit at the sextupole pair, the sextupole pair of the chromaticity correction is effectively worked as a quadrupole. These sextupole bumps are used to correct the optics distortion localized into the arc section.

Figure 1 shows an example of the beta measurement before and after correction. At the first measurement before correction, the relative error of the beta function $\triangle \beta / \beta$ exceeds $15 \%$ in rms and the betatron tune shift exceeding $5 \times 10^{-3}$ is observed. At the measurement after two global-beta correction, the relative error of the beta function is converged into $5 \%$ in rms and the betatron tune shift is corrected into $5 \times 10^{-4}$. The interval between these two measurement is about 24 minutes that includes a XY coupling correction and a dispersion correction procedure.

\section{SUMMARY}

The global-beta measurement and correction presented in previous sections is used as a part of the optics correction of the KEKB ring startup process. It is very helpful to operate the KEKB rings nearby the half-integer resonance line.

\section{REFERENCES}

[1] KEKB Accelerator papers, NIM Section A, Vol. 499, Issue 1,p. 1-233(2003)

[2] Strategic Accelerator Design, http://acc-physics.kek.jp/SAD/

[3] Experimental Physics and Industrial Control System, http://www.aps.anl.gov/epics/

[4] Tool Command Language and Tk GUI toolkit, http://www.tcl.tk/software/tcltk/

[5] KEKB B-Factory Design Report, KEK Report 95-7(1995), http://www-acc.kek.jp/kekb/publication/

KEKB_design_report/KEKB\%20Design\%20Report.html 